\title{
Correction to: Evolutionary Games for Audiovisual Works: Exploring the Demographic Prisoner's Dilemma
}

\author{
Stefano Kalonaris (D)
}

\section{Correction to: \\ Chapter "Evolutionary Games for Audiovisual Works: \\ Exploring the Demographic Prisoner's Dilemma" \\ in: A. Ekárt et al. (Eds.): Computational Intelligence in Music, Sound, Art and Design, LNCS 11453, https://doi.org/10.1007/978-3-030-16667-0_7}

In an older version of this paper, a footnote was included at the bottom of page 102 . This was removed as it no longer linked to the page that it was intended to link to. 